Publ. Mat. 62 (2018), 75-94

DOI: 10.5565 /PUBLMAT6211804

\title{
WEIGHTED SQUARE FUNCTION INEQUALITIES
}

\author{
ADAM OSȨKOWSKI
}

\begin{abstract}
For an integrable function $f$ on $[0,1)^{d}$, let $S(f)$ and $M f$ denote the corresponding dyadic square function and the dyadic maximal function of $f$, respectively. The paper contains the proofs of the following statements.
\end{abstract}

(i) If $w$ is a dyadic $A_{1}$ weight on $[0,1)^{d}$, then

$$
\|S(f)\|_{L^{1}(w)} \leq \sqrt{5}[w]_{A_{1}}^{1 / 2}\|M f\|_{L^{1}(w)} .
$$

The exponent $1 / 2$ is shown to be the best possible.

(ii) For any $p>1$, there are no constants $c_{p}, \alpha_{p}$ depending only on $p$ such that for all dyadic $A_{p}$ weights $w$ on $[0,1)^{d}$,

$$
\|S(f)\|_{L^{1}(w)} \leq c_{p}[w]_{A_{p}}^{\alpha_{p}}\|M f\|_{L^{1}(w)} .
$$

2010 Mathematics Subject Classification: Primary: 42B25; Secondary: 46E30, $60 \mathrm{G} 42$.

Key words: Square function, maximal operator, dyadic, weight, Bellman function.

\section{Introduction}

As evidenced in numerous works (see e.g. Carlen and Krée [4], Dellacherie and Meyer [7], Pisier and Xu [21], Stein [22, 23]), square function inequalities have played an important role in many areas of mathematics. In particular, it is often of interest to obtain optimal, or at least good bounds for the constants involved in such estimates. The purpose of this paper is to study tight weighted versions of Davis' inequality, which compares the $L^{1}$ norms of a dyadic square function and a dyadic maximal function, with a constant independent of the dimension.

Let us start with introducing the necessary background and notation. Throughout the paper, we will work with the unit cube $[0,1)^{d}$, equipped with its dyadic subcubes, i.e., sets of the form $\left[\frac{a_{1}}{2^{n}}, \frac{a_{1}+1}{2^{n}}\right) \times\left[\frac{a_{2}}{2^{n}}, \frac{a_{2}+1}{2^{n}}\right) \times$ $\cdots \times\left[\frac{a_{d}}{2^{n}}, \frac{a_{d}+1}{2^{n}}\right)$, where $n$ is some nonnegative integer and $a_{1}, a_{2}, \ldots, a_{d}$

Research supported by the National Science Centre, Poland, grant DEC-2014/14/E/ ST1/00532. 
are some elements of $\left\{0,1, \ldots, 2^{n}-1\right\}$. For $n \geq 0$, let $\mathcal{Q}^{n}$ denote the collection of all dyadic cubes of measure $2^{-n d}$ contained in $[0,1)^{d}$. Suppose that $f:[0,1)^{d} \rightarrow \mathbb{R}$ is a fixed integrable function. Given a dyadic cube $Q$, we will denote by $\langle f\rangle_{Q}$ the average of $f$ over $Q$, i.e., $\langle f\rangle_{Q}=\frac{1}{|Q|} \int_{Q} f$. For any $n \geq 0$, we define $f_{n}$ to be the conditional expectation of $f$ with respect to $\mathcal{Q}^{n}$, i.e., a function on $[0,1)^{d}$, which is constant on each element of $\mathcal{Q}^{n}$ and such that $\langle f\rangle_{Q}=\left\langle f_{n}\right\rangle_{Q}$ for any $Q \in \mathcal{Q}^{n}$; in other words,

$$
f_{n}=\sum_{Q \in \mathcal{Q}^{n}}\langle f\rangle_{Q} \chi_{Q} .
$$

In a probabilistic language, this amounts to saying that $\left(f_{n}\right)_{n \geq 0}$ is the martingale associated with $f$, adapted to the dyadic filtration. We define the corresponding difference sequence $\left(d f_{n}\right)_{n \geq 0}$ by putting $d f_{0}=f_{0}$ and $d f_{n}=f_{n}-f_{n-1}, n=1,2, \ldots$ Next, we introduce the dyadic square function $S(f)$ and the dyadic maximal function $M f$ by the formulae

$$
S(f)=\left(\sum_{k=0}^{\infty}\left|d f_{k}\right|^{2}\right)^{1 / 2} \text { and } M f=\sup _{k \geq 0}\left|f_{k}\right| .
$$

We will also use the notation

$$
S_{n}(f):=S\left(f_{n}\right)=\left(\sum_{k=0}^{n}\left|d f_{k}\right|^{2}\right)^{1 / 2} \quad \text { and } \quad M_{n} f:=M\left(f_{n}\right)=\max _{0 \leq k \leq n}\left|f_{k}\right|
$$

for $n=0,1,2, \ldots$

The problem of comparing various sizes of $f, S(f)$, and $M f$ is classical and goes back to the works of Khintchine [11], Littlewood [14], Paley [19], Marcinkiewicz [15], and Marcinkiewicz and Zygmund [16]. Our motivation comes from the moment estimates

$$
\|S(f)\|_{L^{p}} \leq C_{p}\|f\|_{L^{p}}, \quad 1<p<\infty,
$$

where the constants $C_{p}$ depend only on $p$ (and not on the dimension). This inequality was established by Burkholder in [2], actually in a slightly more general setting of martingales. In the boundary case $p=1$, the inequality does not hold with any finite constant. However, as Davis showed in $[6]$, we have the substitute

$$
\|S(f)\|_{L^{1}} \leq C_{1}\|M f\|_{L^{1}}
$$

with some universal $C_{1}<\infty$. Burkholder [3] proved that $C_{1}=\sqrt{3}$ is optimal.

In this paper we will be interested in a weighted version of (1.2). Suppose that $w$ is a weight, i.e., a positive, integrable function on $[0,1)^{d}$. 
Given $1<p<\infty$, we say that $w$ is a dyadic $A_{p}$ weight if it satisfies the dyadic analogue of Muckenhoupt's condition:

$$
[w]_{A_{p}}:=\sup \left(\frac{1}{|Q|} \int_{Q} w(x) \mathrm{d} x\right)\left(\frac{1}{|Q|} \int_{Q} w(x)^{-1 /(p-1)} \mathrm{d} x\right)^{p-1}<\infty,
$$

where the supremum is taken over all dyadic cubes contained in $[0,1)^{d}$. Sometimes, to stress that we consider weights on $[0,1)^{d}$, one uses the notation $A_{p}\left([0,1)^{d}\right)$. Furthermore, $w$ is a dyadic $A_{\infty}$ weight, if

$$
[w]_{A_{\infty}}:=\sup \left(\frac{1}{|Q|} \int_{Q} w(x) \mathrm{d} x\right) \exp \left(-\frac{1}{|Q|} \int_{Q} \log w(x) \mathrm{d} x\right)<\infty,
$$

where the supremum is taken over the same set as above. Finally, $w$ is a dyadic $A_{1}$ weight if there is a constant $C$ depending only on $w$ such that for any dyadic cube $Q \subseteq[0,1)^{d}$,

$$
\frac{1}{|Q|} \int_{Q} w(x) \mathrm{d} x \leq \underset{Q}{\operatorname{essinf}} w .
$$

The smallest $C$ with this property is denoted by $[w]_{A_{1}}$; observe that $[w]_{A_{1}} \geq 1$. It follows directly from Hölder's inequality that $A_{p}$ classes grow as $p$ increases. Furthermore, it is well-known that $A_{\infty}=\cup_{1 \leq p<\infty} A_{p}$.

We come back to weighted square function inequalities, and we will be interested in obtaining tight bounds for the constants involved. Buckley [1] showed the $L^{2}$ inequality

$$
\|S(f)\|_{L^{2}(w)} \leq C[w]_{A_{2}}^{3 / 2}\|f\|_{L^{2}(w)},
$$

with $C$ being a universal constant. This is probably the first result in the literature, which singled out the dependence of the constant on the $A_{p}$ characteristics of the weight. This gave rise to the question about the optimal (i.e., the smallest possible) exponent at $[w]_{A_{2}}$ in (1.3). This problem remained open for a few years and was finally answered by Hukovič, Treil, and Volberg [10]: the exponent 1 is the best. See also the paper [20] by Petermichl and Pott, and [26] by Wittwer. This result was significantly extended by Cruz-Uribe, Martell, and Pérez [5] to the case of general $A_{p}$ weights. Here is the precise statement.

Theorem 1.1. Let $1<p<\infty$. Then there is a constant $C=C_{p, d}$ depending only on the parameters indicated such that for any $A_{p}$ weight $w$ and any integrable function $f$,

$$
\|S(f)\|_{L^{p}(w)} \leq C_{p, d}[w]_{A_{p}}^{\max \{1 / 2,1 /(p-1)\}}\|f\|_{L^{p}(w)} .
$$

The exponent $\max \{1 / 2,1 /(p-1)\}$ is the best possible, i.e., it cannot be replaced in general by a smaller number. 
Consult also the works of Gundy and Wheeden [9], Dragičević et al. [8] and Lerner $[\mathbf{1 2}, \mathbf{1 3}]$ for related results. See also the book by Wilson [25] for an overview of weighted square function inequalities.

We turn our attention to the description of the results of this paper. In the first theorem, we provide the substitute of (1.4) in the case $p=1$. Actually, we will work in a more general, vector setting. Let $\mathcal{H}$ be a separable Hilbert space with scalar product $\langle\cdot, \cdot\rangle$ and a norm $|\cdot|$, and let $f:[0,1)^{d} \rightarrow \mathcal{H}$ be an integrable function. Then the above definitions of $S(f)$ and $M f$ make perfect sense and hence we can ask about the comparison of weighted $L^{1}$ norms of $S(f)$ and $M f$. Here is the answer.

Theorem 1.2. If $w$ is an $A_{1}$ weight, then

$$
\|S(f)\|_{L^{1}(w)} \leq \sqrt{5}[w]_{A_{1}}^{1 / 2}\|M f\|_{L^{1}(w)}
$$

for any integrable $f:[0,1)^{d} \rightarrow \mathcal{H}$. The exponent $1 / 2$ cannot be replaced by a smaller number.

The above result can be regarded as a uniform maximal estimate for the square function: here by uniformity we mean that the multiplicative constant does not depend on the dimension. Actually, this constant is not far from optimal: if $w \equiv 1$, then the best choice is $\sqrt{3}$, as we have noted above (cf. [3]).

In the next theorem, we study the $A_{p}$ version of Theorem 1.2.

Theorem 1.3. Let $p>1$ and let $K$ be an arbitrary positive constant. Then there is a positive integer $d$, an integrable function $f:[0,1)^{d} \rightarrow \mathbb{R}$, and an $A_{p}$ weight $w$ on $[0,1)^{d}$ satisfying $[w]_{A_{p}} \leq 2$, such that

$$
\|S(f)\|_{L^{1}(w)}>K\|M f\|_{L^{1}(w)} .
$$

So, there is no uniform square function bound for $A_{p}$ weights, even if $f$ is assumed to be real-valued. What if we allow the dependence of the constant on $d$ ? Then the corresponding bound holds even for the $A_{\infty}$ class. Here is the classical result, proved by Gundy and Wheeden [9] in the one-dimensional case (however, the reasoning presented there extends easily to general $d$ ).

Theorem 1.4. Let $w$ be an $A_{\infty}$ weight on $[0,1)^{d}$. Then there is a constant $C=C\left(d,[w]_{A_{\infty}}\right)$ depending on the parameters indicated such that

$$
\|S(f)\|_{L^{1}(w)} \leq C\|M f\|_{L^{1}(w)}
$$

for any integrable function $f$ on $[0,1)^{d}$. 
A few words about the proof and the organization of the paper are in order. The next section is devoted to the proof of Theorem 1.2. We establish the inequality (1.5) with the use of the so-called Bellman function method, a powerful technique used widely in analysis and probability (cf. [17], [18], and the paper [10] already cited above). The sharpness of the exponent $1 / 2$ is shown with the use of appropriate examples. Section 3 is devoted to Theorem 1.3, which is again proved with the use of Bellman function method.

\section{Proof of Theorem 1.2}

We have decided to split this section into four separate parts.

2.1. On the method of proof. Let us describe the technique, which will be used to establish the inequality (1.5). First, it is instructive to recall the reasoning of Burkholder [3], which allowed him to obtain (1.2) with $C_{1}=\sqrt{3}$. Burkholder introduced the special function $\mathcal{U}: \mathcal{H} \times$ $[0, \infty) \times(0, \infty) \rightarrow \mathbb{R}$, given by

$$
\mathcal{U}(x, y, z)=\frac{y^{2}-|x|^{2}-2(|x| \vee z)^{2}}{|x| \vee z}
$$

(here and below, $a \vee b=\max \{a, b\}$ ) and proved that it enjoys the following three properties. First, for any $x \in \mathcal{H} \backslash\{0\}$,

$$
\mathcal{U}(x,|x|,|x|) \leq 0 .
$$

Second, for any $(x, y, z) \in \mathcal{H} \times[0, \infty) \times(0, \infty)$, we have the majorization

$$
\mathcal{U}(x, y, z) \geq 2 \sqrt{3}(y-\sqrt{3}(|x| \vee z)) .
$$

The final property is the following: if $(x, y, z) \in \mathcal{H} \times[0, \infty) \times(0, \infty)$, $Q \subset[0,1)^{d}$ is a dyadic cube and $h: Q \rightarrow \mathbb{R}$ is any simple function of average 0 , then

$$
\frac{1}{|Q|} \int_{Q} \mathcal{U}\left(x+h(s), \sqrt{y^{2}+h^{2}(s)}, z\right) \mathrm{d} s \leq \mathcal{U}(x, y, z) .
$$

Having checked these conditions, inequality (1.2) follows quickly. Namely, it is not difficult to show that the third property above is actually equivalent to showing that for any function $f$ the sequence

$$
\left(\int_{[0,1)^{d}} \mathcal{U}\left(f_{n}, S_{n}(f), M f_{n}\right) \mathrm{d} s\right)_{n \geq 0}
$$


is nonincreasing. Combining this with the above majorization yields

$$
\begin{aligned}
2 \sqrt{3}\left(\int_{[0,1)^{d}}\left(S_{n}(f)-\sqrt{3} M f_{n}\right) \mathrm{d} s\right) & \leq \int_{[0,1)^{d}} \mathcal{U}\left(f_{n}, S_{n}(f), M f_{n}\right) \mathrm{d} s \\
& \leq \int_{[0,1)^{d}} \mathcal{U}\left(f_{0}, S_{0}(f), M f_{0}\right) \mathrm{d} s .
\end{aligned}
$$

But the latter integrand is nonpositive, due to (2.1), since $\left|f_{0}\right|=S_{0}(f)=$ $M f_{0}$ (we may assume from the very beginning that $f_{0} \neq 0$, by a simple continuity argument). Thus $\left\|S_{n}(f)\right\|_{L^{1}} \leq \sqrt{3}\left\|M f_{n}\right\|_{L^{1}}$ and letting $n \rightarrow$ $\infty$ completes the proof.

Our argument below will follow the same pattern and will exploit majorization and concavity-type properties of an appropriate special function. However, due to the appearance of an $A_{1}$ weight in the estimate, we will be forced to introduce additional two variables. Let us explain this in a more detailed manner. Following [24], each dyadic $A_{1}$ weight $w$ on $[0,1)^{d}$, satisfying $[w]_{A_{1}} \leq c$, gives rise to two functional sequences $\left(w_{n}\right)_{n \geq 0}$ and $\left(v_{n}\right)_{n \geq 0}$, which capture its behavior. Namely, $\left(w_{n}\right)_{n \geq 0}$ is the associated martingale (sequence of conditional expectations of $w)$ and for any $n \geq 0$, the function $v_{n}:[0,1)^{d} \rightarrow[0, \infty)$ is given as follows: if $Q \in \mathcal{Q}^{n}$, then $v_{n}$ is identically $\operatorname{essinf}_{Q} w$ on $Q$.

These objects enjoy the following properties. Suppose that $n \geq 0$ and let $Q$ be an element of $\mathcal{Q}^{n}$. Then, by the very definition, $w_{n}$ and $v_{n}$ are constant on $Q$; furthermore, we have

$$
\frac{1}{|Q|} \int_{Q} w_{n+1}(x) \mathrm{d} x=\left.w_{n}\right|_{Q}, \quad v_{n+1} \geq v_{n}, \quad \text { and } \quad v_{n} \leq w_{n} \leq c v_{n},
$$

where the latter bound follows from the $A_{1}$ condition.

As we have announced in the introductory section, our principal goal is the study of the estimate

$$
\|S(f)\|_{L^{1}(w)} \leq C[w]_{A_{1}}^{\alpha}\|M f\|_{L^{1}(w)},
$$

where $C$ is some numerical constant and the parameter $\alpha$ is as small as possible. Suppose that $w$ is an $A_{1}$ weight on $[0,1)^{d}$, with $[w]_{A_{1}} \leq c$. In the light of Burkholder's proof presented above, it is natural to search for a special function $B$ of five variables, defined on the domain

$$
\mathcal{D}_{c}(B)=\left\{(x, y, z, w, v) \in \mathcal{H} \times[0, \infty)^{4}: x \neq 0, z>0, v \leq w \leq c v\right\},
$$

which enjoys the following three properties. First, if $x \in \mathcal{H} \backslash\{0\}$ and $0<v \leq w \leq c v$, then

$$
B(x,|x|,|x|, w, v) \leq 0 .
$$


Second, there is $\beta>0$ such that for any $(x, y, z, w, v) \in \mathcal{D}_{c}(B)$ we have the majorization

$$
B(x, y, z, w, v) \geq \beta\left(|y| w-C c^{\alpha}(|x| \vee z) w\right) .
$$

Finally, if $f$ is an arbitrary function on $[0,1)^{d}$ and $w$ is an $A_{1}$ weight with $[w]_{A_{1}} \leq c$, then the sequence

$$
\left(\int_{[0,1)^{d}} B\left(f_{n}, S_{n}(f), M f_{n}, w_{n}, v_{n}\right) \mathrm{d} s\right)_{n \geq 0}
$$

is nonincreasing. How to find an appropriate $B$ ? Clearly, we expect that if we plug $w=v \equiv 1$ (which corresponds to the unweighted setting), then $B(\cdot, \cdot, \cdot, w, v)$ should be equal, or at least "close" to Burkholder's function $\mathcal{U}$ discussed above. Combining this observation with (2.3) suggests considering the function

$$
B(x, y, z, w, v)=\frac{y^{2} w-\gamma_{1} c^{2 \alpha}|x|^{2} w-\gamma_{2} c^{2 \alpha}(|x| \vee z)^{2} w}{|x| \vee z},
$$

where $\gamma_{1}, \gamma_{2}$ are some positive constants. However, then the third requirement is not satisfied, as one easily verifies; furthermore, the variable $v$ does not appear in the formula which indicates that $B$ might not control the full $A_{1}$ property of the weight. A little thought and experimentation leads to the choice

$$
B(x, y, z, w, v)=\frac{y^{2} w-\gamma_{1} c^{2 \alpha}|x|^{2} v-\gamma_{2} c^{2 \alpha}(|x| \vee z)^{2} v}{|x| \vee z},
$$

which works with $\alpha=1 / 2$ and $\gamma_{1}=1, \gamma_{2}=4$. See below for details.

2.2. A special function and some auxiliary facts. For a fixed $c \geq$ 1 , let $B=B_{c}: \mathcal{D}_{c}(B) \rightarrow \mathbb{R}$ be given by the formula

$$
B(x, y, z, w, v)=\frac{y^{2} w-c|x|^{2} v-4 c(|x| \vee z)^{2} v}{|x| \vee z} .
$$

As we have already discussed above, we will require appropriate majorizations.

Lemma 2.1. (i) If $x \in \mathcal{H} \backslash\{0\}$ and $0<v \leq w \leq c v$, then (2.2) holds.

(ii) For any $(x, y, z, w, v) \in \mathcal{D}_{c}(B)$ we have

$$
B(x, y, z, w, v) \geq 2 \sqrt{5 c}(y w-\sqrt{5 c}(|x| \vee z) w) .
$$

Proof: The first property is trivial, since $y^{2} w-c|x|^{2} v \leq 0$. In the proof of (2.4) we may assume that $|x| \vee z=1$, since $B(\lambda x, \lambda y, \lambda z, w, v)=$ $\lambda B(x, y, z, w, v)$ for any $\lambda>0$. It suffices to note that

$$
y^{2} w-c|x|^{2} v-4 c v \geq y^{2} w-5 c w \geq 2 \sqrt{5 c}(y w-\sqrt{5 c} w),
$$

which is the desired estimate. 
2.3. Proof of (1.5). Set $c=[w]_{A_{1}}$ and let $B=B_{c}$ be the function introduced in the previous subsection. Without loss of generality, we may assume that $f_{0} \neq 0$.

The first step is to show that for each $n \geq 0$,

$$
\begin{aligned}
\int_{[0,1)^{d}} B\left(f_{n+1}, S_{n+1}(f),\right. & \left.M f_{n+1}, w_{n+1}, v_{n+1}\right) \mathrm{d} x \\
\leq & \int_{[0,1)^{d}} B\left(f_{n}, S_{n}(f), M f_{n}, w_{n}, v_{n}\right) \mathrm{d} x,
\end{aligned}
$$

the monotonicity we discussed above. To do this, fix an element $Q$ of $\mathcal{Q}^{n}$. We will often use that $f_{n}, S_{n}(f), M f_{n}, w_{n}$, and $v_{n}$ are constant on $Q$. We have $M f_{n} \leq M f_{n+1}$ and $w_{n+1} \leq c v_{n+1}$, so

$$
\begin{aligned}
\int_{Q} \frac{S_{n+1}^{2}(f) w_{n+1}}{M f_{n+1}} \mathrm{~d} x & \leq \int_{Q} \frac{S_{n}^{2}(f) w_{n+1}}{M f_{n}} \mathrm{~d} x+c \int_{Q} \frac{\left|d f_{n+1}\right|^{2} v_{n+1}}{M f_{n+1}} \mathrm{~d} x \\
& =\int_{Q} \frac{S_{n}^{2}(f) w_{n}}{M f_{n}} \mathrm{~d} x+c \int_{Q} \frac{\left|d f_{n+1}\right|^{2} v_{n+1}}{M f_{n+1}} \mathrm{~d} x
\end{aligned}
$$

where in the second passage we have used the fact that $w_{n}$ and $w_{n+1}$ have the same average over $Q$. Next, write

$$
\begin{aligned}
& \int_{Q} \frac{-c\left|f_{n+1}\right|^{2} v_{n+1}-4 c\left(M f_{n+1}\right)^{2} v_{n+1}}{M f_{n+1}} \mathrm{~d} x \\
& =-c \int_{Q} \frac{\left|d f_{n+1}\right|^{2} v_{n+1}+\left(\left|f_{n}\right|^{2}+2\left\langle f_{n}, d f_{n+1}\right\rangle+4\left(M f_{n+1}\right)^{2}\right) v_{n+1}}{M f_{n+1}} \mathrm{~d} x .
\end{aligned}
$$

Recall that $v_{n+1} \geq v_{n}$; furthermore, we have

$$
2\left\langle f_{n}, d f_{n+1}\right\rangle \geq-2\left|f_{n}\right|\left|d f_{n+1}\right|=-2\left|f_{n}\right|\left|f_{n+1}-f_{n}\right| \geq-4\left(M f_{n+1}\right)^{2} .
$$

Consequently, the expression on the right in (2.7) can be bounded from above by

$$
-c \int_{Q}\left(\frac{\left|d f_{n+1}\right|^{2} v_{n+1}}{M f_{n+1}}+\frac{\left(\left|f_{n}\right|^{2}+2\left\langle f_{n}, d f_{n+1}\right\rangle+4\left(M f_{n+1}\right)^{2}\right) v_{n}}{M f_{n+1}}\right) \mathrm{d} x .
$$

Combining this with (2.6), we obtain

$$
\begin{aligned}
& \int_{Q} B\left(f_{n+1}, S_{n+1}(f), M f_{n+1}, w_{n+1}, v_{n+1}\right) \mathrm{d} x \\
& \leq \int_{Q}\left(\frac{S_{n}^{2}(f) w_{n}}{M f_{n}}-\frac{c\left(\left|f_{n}\right|^{2}+2\left\langle f_{n}, d f_{n+1}\right\rangle+4\left(M f_{n+1}\right)^{2}\right) v_{n}}{M f_{n+1}}\right) \mathrm{d} x .
\end{aligned}
$$


Now we will prove that

$$
\begin{aligned}
& \frac{\left(\left|f_{n}\right|^{2}+2\left\langle f_{n}, d f_{n+1}\right\rangle+4\left(M f_{n+1}\right)^{2}\right) v_{n}}{M f_{n+1}} \\
& \geq \frac{\left(\left|f_{n}\right|^{2}+2\left\langle f_{n}, d f_{n+1}\right\rangle+4\left(M f_{n}\right)^{2}\right) v_{n}}{M f_{n}},
\end{aligned}
$$

which is equivalent to

$$
l\left(\left|f_{n}\right|^{2}+2\left\langle f_{n}, d f_{n+1}\right\rangle-4 M f_{n} M f_{n+1}\right)\left(M f_{n+1}-M f_{n}\right) v_{n} \leq 0 .
$$

This is true: we have $M f_{n+1} \geq M f_{n}, v_{n} \geq 0$ and

$$
\begin{aligned}
\left|f_{n}\right|^{2}+2\left\langle f_{n}, d f_{n+1}\right\rangle & =\left\langle f_{n}, f_{n+1}\right\rangle+\left\langle f_{n}, d f_{n+1}\right\rangle \\
& \leq M f_{n} M f_{n+1}+2 M f_{n} M f_{n+1}<4 M f_{n} M f_{n+1} .
\end{aligned}
$$

Hence, coming back to (2.8), we see that we have established the bound

$$
\begin{aligned}
\int_{Q} B & \left(f_{n+1}, S_{n+1}(f), M f_{n+1}, w_{n+1}, v_{n+1}\right) \mathrm{d} x \\
& \leq \int_{Q} B\left(f_{n}, S_{n}(f), M f_{n}, w_{n}, v_{n}\right) \mathrm{d} x-2 c \int_{Q} \frac{\left\langle f_{n}, d f_{n+1}\right\rangle v_{n}}{M f_{n}} \mathrm{~d} x \\
& =\int_{Q} B\left(f_{n}, S_{n}(f), M f_{n}, w_{n}, v_{n}\right) \mathrm{d} x,
\end{aligned}
$$

where in the last line we have exploited the equality $\int_{Q} d f_{n+1} \mathrm{~d} x=0$ and the fact that $f_{n}, v_{n}$, and $M f_{n}$ are constant on $Q$. Summing over all $Q \in \mathcal{Q}^{n}$, we get the desired monotonicity property $(2.5)$.

The next step is to exploit (2.2) and then (2.4), to get

$$
\begin{aligned}
0 & \geq \int_{[0,1]^{d}} B\left(f_{0}, S_{0}(f), M f_{0}, w_{0}, v_{0}\right) \mathrm{d} x \\
& \geq \int_{[0,1]^{d}} B\left(f_{n}, S_{n}(f), M f_{n}, w_{n}, v_{n}\right) \mathrm{d} x \\
& \geq 2 \sqrt{5 c} \int_{[0,1]^{d}}\left(S_{n}(f) w_{n}-\sqrt{5 c} M f_{n} w_{n}\right) \mathrm{d} x .
\end{aligned}
$$

Consequently, we have proved that

$$
\int_{[0,1]^{d}} S_{n}(f) w_{n} \mathrm{~d} x \leq \sqrt{5 c} \int_{[0,1]^{d}} M f_{n} w_{n} \mathrm{~d} x
$$


and hence

$$
\int_{[0,1]^{d}} S_{n}(f) w \mathrm{~d} x \leq \sqrt{5 c} \int_{[0,1]^{d}} M f_{n} w \mathrm{~d} x,
$$

since $w_{n}$ is the conditional expectation of $w$ with respect to $\mathcal{Q}^{n}$. It remains to let $n \rightarrow \infty$ and apply Lebesgue's monotone convergence theorem.

2.4. Sharpness of the exponent. Let $N$ be a fixed nonnegative integer and let $Q^{n}=\left[0,2^{-n}\right)^{d}$ for $n=0,1,2, \ldots$ Choose $c \geq 1$ and define $w:[0,1)^{d} \rightarrow[0, \infty)$ by

$w(x)= \begin{cases}\left(1+\left(2^{d}-1\right)\left(1-c^{-1}\right)\right)^{n} c^{-1} & \text { if } x \in Q^{n} \backslash Q^{n+1}, 0 \leq n \leq N-1, \\ l\left(1+\left(2^{d}-1\right)\left(1-c^{-1}\right)\right)^{N} & \text { if } x \in Q^{N} .\end{cases}$

Note that $w$ is an $A_{1}$-weight and $[w]_{A_{1}}=c$. To check this, pick an arbitrary dyadic cube $Q$. If $Q$ is contained in $Q^{N}$ or the two cubes are disjoint, then $w$ is constant on $Q$, so $\operatorname{essinf}_{Q} w=|Q|^{-1} \int_{Q} w$ and the desired inequality is satisfied. It remains to consider the case when $Q$ contains $Q^{N}$ (and $\left.Q \neq Q^{N}\right)$. Then we have $Q=Q^{k}$ for some $k=$ $0,1,2, \ldots, N-1$ and

$$
\underset{Q}{\operatorname{essinf}} w=\left(1+\left(2^{d}-1\right)\left(1-c^{-1}\right)\right)^{k} c^{-1}=c^{-1} \cdot \frac{1}{|Q|} \int_{Q} w,
$$

so the requirement is met. Hence $w \in A_{1}$ and, as a by-product, we see that $\int_{[0,1)^{d}} w=\int_{Q^{0}} w=1$.

Next, consider a sequence $\left(d f_{n}\right)_{n=0}^{N}$ of functions, given by $d f_{0}=\chi_{[0,1)^{d}}$ and

$$
d f_{n}=(-1)^{n}\left(2 \chi_{\left[0,2^{-n+1}\right)^{d} \backslash\left[2^{-n}, 2^{-n+1}\right)^{d}}-\left(2^{d+1}-2\right) \chi_{\left[2^{-n}, 2^{-n+1}\right)^{d}}\right),
$$

for $n=1,2, \ldots, N$. Note that $d f_{n}$ is measurable with respect to the $\sigma$ algebra generated by $\mathcal{Q}^{n}$. Furthermore, if $n \geq 1$, then for each $Q \in \mathcal{Q}^{n-1}$ we have $\int_{Q} d f_{n} \mathrm{~d} x=0$. Consequently, if we define $f=d f_{0}+d f_{1}+d f_{2}+$ $\cdots+d f_{N}$, then for each $n=0,1,2, \ldots, N$ we have $f_{n}=d f_{0}+d f_{1}+\cdots+d f_{n}$.

To gain some intuition about the behavior of $f$ and $M f$, pick $x \in$ $[0,1)^{d}$. If $x$ belongs to the cube $\left[2^{-n}, 2^{-n+1}\right)^{d}$ for some $n=1,2, \ldots, N$, then we easily check that $f_{k}(x)=(-1)^{k}$ for $0 \leq k \leq n-1$ and $f_{k}(x)=$ $(-1)^{n-1}\left(2^{d+1}-1\right)$ for $k \geq n$, and hence $M f(x)=2^{d+1}-1$. For remaining $x \in[0,1)^{d}$, it is not difficult to see that the sequence $f_{0}, f_{1}, f_{2}, \ldots, f_{n}$ is an alternating sequence of signs, which stabilizes after some number of 
steps (and hence $M f(x)=1$ ). Consequently, we may write

$$
\begin{aligned}
\int_{[0,1)^{d}} M f(x) w(x) \mathrm{d} x & =\int_{[0,1)^{d}} w(x) \mathrm{d} x+\sum_{n=1}^{N}\left(2^{d+1}-2\right) \int_{\left[2^{-n}, 2^{-n+1}\right)^{d}} w(x) \mathrm{d} x \\
& =1+\sum_{n=1}^{N}\left(2^{d+1}-2\right) 2^{-n d}\left(1+\left(2^{d}-1\right)\left(1-c^{-1}\right)\right)^{n-1} c^{-1} \\
& =1+2\left(1-\left(1-\left(1-2^{-d}\right) c^{-1}\right)^{N}\right) .
\end{aligned}
$$

To analyze the square function, note that for $x \in\left[0,2^{-N}\right)^{d}$ we have $d f_{0}(x)=1$ and $\left|d f_{n}(x)\right|=2,1 \leq n \leq N$. This implies $S f(x)=$ $S_{N} f(x)=(1+4 N)^{1 / 2}$ and hence

$$
\begin{aligned}
\int_{[0,1)^{d}} S(f)(x) w(x) \mathrm{d} x & \geq \int_{\left[0,2^{-N}\right)^{d}} S(f)(x) w(x) \mathrm{d} x \\
& =(1+4 N)^{1 / 2}\left(1-\left(1-2^{-d}\right) c^{-1}\right)^{N} .
\end{aligned}
$$

Now take $\alpha<\beta<1 / 2$ and put $N=\left\lfloor c^{2 \beta}\right\rfloor$. Then $\lim _{c \rightarrow \infty}(1-(1-$ $\left.\left.2^{-d}\right) c^{-1}\right)^{N}=1$ and hence, by the above calculations,

$$
\frac{\int_{[0,1)^{d}} S(f)(x) w(x) \mathrm{d} x}{[w]_{A_{1}}^{\alpha} \int_{[0,1)^{d}} M f(x) w(x) \mathrm{d} x}=O\left(c^{\beta-\alpha}\right), \quad \text { as } c \rightarrow \infty .
$$

Letting $c \rightarrow \infty$, we see that no exponent $\alpha<1 / 2$ suffices in (1.5).

\section{Lack of estimates for $A_{p}$ weights, $p>1$}

Now we take a look at the uniform weighted bounds between $\|S(f)\|_{L^{1}(w)}$ and $\|M f\|_{L^{1}(w)}$ when $w$ is assumed to be an $A_{p}$ weight, $p>1$. Suppose, contrary to the assertion of Theorem 1.3, that there is $1<p<\infty$ and a constant $K$ depending only on $p$, such that for any dimension $d$, any function $f$ on $[0,1)^{d}$ and any $A_{p}$ weight $w$ on $[0,1)^{d}$ with $[w]_{A_{p}} \leq 2$, we have

$$
\|S(f)\|_{L^{1}(w)} \leq K\|M f\|_{L^{1}(w)} .
$$

Fix an arbitrary integer $N$. The appropriate examples have quite complicated, fractal-type structure. To avoid the technicalities which arise in their study, we will follow a different approach. In a sense, it can be regarded as a dual to the approach presented in the previous section. Namely, we will show that the validity of (3.1) implies the existence of 
a certain abstract function, possessing appropriate concavity and enjoying a certain majorization. Then, by exploiting these properties in an appropriate order, we will obtain a contradiction.

We start with an auxiliary geometrical fact.

Lemma 3.1. Let $p, N$, and $d$ be as above.

(i) Let $\boldsymbol{w}, \boldsymbol{v}$ be two positive numbers satisfying $\boldsymbol{w}^{p-1} \geq 1$. Then there are points $P, Q$ lying on the curve $x y^{p-1}=1$ such that $P+Q=(2 \boldsymbol{w}, 2 \boldsymbol{v})$.

(ii) Suppose that $\boldsymbol{w}=1$ and $\boldsymbol{v}=2^{1 /(p-1)}$. Then there are two points $R, T$ such that $R=\left(R_{x}, R_{y}\right)$ lies on the curve $x y^{p-1}=2, T=\left(T_{x}, T_{y}\right)$ lies on the curve $x y^{p-1}=1, R_{x} \leq T_{x}$, and

$$
\left(1-\left(1-2^{-d}\right)^{N}\right) R+\left(1-2^{-d}\right)^{N} T=(\boldsymbol{w}, \boldsymbol{v}) .
$$

Furthermore,

$$
\left(1-\left(1-2^{-d}\right)^{N}\right) 2^{d} R_{x}<1
$$

provided $d$ is sufficiently large.

Proof: (i) The argument is very simple. Pick any point $P=\left(P_{x}, P_{y}\right)$ on the curve $x y^{p-1}=1$ and let $Q$ be defined by the condition $(P+Q) / 2=$ $(\mathbf{w}, \mathbf{v})$. Then $Q$ is a continuous function of $P$. Furthermore, if $P_{y}$ is huge, then $Q_{y}$ is negative, so $Q$ lies below the curve $x y^{p-1}=1$. On the other hand, when $P_{y}=\mathbf{v}$, then $P_{x}=P_{y}^{1-p}=\mathbf{v}^{1-p} \leq \mathbf{w}$; this implies $Q_{x} \geq \mathbf{w}$ and $Q_{y}=\mathbf{v}$, so $Q$ lies on or above the curve. Thus, by Darboux property, there must be a point $P$ for which the desired configuration is satisfied.

(ii) The existence of the points $R, T$ is proved similarly (one analyzes the move of a point $R$ along the curve $x y^{p-1}=2$ ). To show (3.3), we exploit (3.2). We have

$$
1=\left(1-\left(1-2^{-d}\right)^{N}\right) R_{x}+\left(1-2^{-d}\right)^{N} T_{x}
$$

and since $R_{x}<1<T_{x}$,

$$
\begin{aligned}
2^{1 /(p-1)} & =\left(1-\left(1-2^{-d}\right)^{N}\right)\left(\frac{2}{R_{x}}\right)^{1 /(p-1)}+\left(1-2^{-d}\right)^{N} T_{x}^{-1 /(p-1)} \\
& <\left(1-\left(1-2^{-d}\right)^{N}\right)\left(\frac{2}{R_{x}}\right)^{1 /(p-1)}+\left(1-2^{-d}\right)^{N},
\end{aligned}
$$

which implies

$$
R_{x}<\left(\frac{1-\left(1-2^{-d}\right)^{N}}{1-\left(1-2^{-d}\right)^{N} / 2^{1 /(p-1)}}\right)^{p-1}
$$


Thus, if $d \rightarrow \infty$, then $R_{x} \rightarrow 0$; on the other hand, we have (1- (1$\left.\left.2^{-d}\right)^{N}\right) 2^{d} \leq N$ for each $d$. This proves the assertion.

Let $\mathbf{w}, \mathbf{v}, R$, and $T$ be as in (ii). In what follows, we will also exploit the points $T_{0}, T_{1}, \ldots, T_{N}$ given by $T_{0}=(\mathbf{w}, \mathbf{v})$ and the recursive equation

$$
T_{k}=\left(1-2^{-d}\right) T_{k+1}+2^{-d} R .
$$

By straightforward induction, we see that $(\mathbf{w}, \mathbf{v})=\left(1-2^{-d}\right)^{k} T_{k}+(1-$ $\left.\left(1-2^{-d}\right)^{k}\right) R$ for each $k$ and hence in particular $T_{N}=T$.

Now, let $\mathcal{D}$ be a set which consists of all points $(\mathbf{w}, \mathbf{v})$ such that $\mathbf{w}, \mathbf{v}>0,1 \leq \mathbf{w v}^{p-1} \leq 2$. Let $Q$ be a dyadic cube contained in $[0,1)^{d}$. For any $\mathbf{x} \in \mathbb{R}, \mathbf{y} \geq 0, \mathbf{z} \geq 0$, and any $(\mathbf{w}, \mathbf{v}) \in \mathcal{D}$, consider the abstract expression

$$
\begin{aligned}
U(\mathbf{x}, \mathbf{y}, \mathbf{z}, \mathbf{w}, \mathbf{v})=\sup \left\{\frac { 1 } { | Q | } \int _ { Q } \left[\left(\mathbf{y}^{2}-\mathbf{x}^{2}+S^{2}(f)(x)\right)^{1 / 2}\right.\right. \\
-K(M f(x) \vee \mathbf{z})] w(x) \mathrm{d} x\} .
\end{aligned}
$$

Here $K$ is the number appearing in (3.1) and the supremum is taken over all integrable functions $f: Q \rightarrow \mathbb{R}$ with average $\langle f\rangle_{Q}=\mathbf{x}$ and all weights $w \in A_{p}(Q)$ satisfying $[w]_{A_{p}} \leq 2,\langle w\rangle_{Q}=\mathbf{w},\left\langle w^{-1 /(p-1)}\right\rangle_{Q}=\mathbf{v}$.

Before we proceed, we need to make two comments. First, we need to show that for each $\mathbf{w}, \mathbf{v}$ as above, at least one weight satisfying appropriate conditions exists: this will guarantee that $U$ is well-defined. To prove this existence, split the cube $Q$ into two halves and put $w=P_{x}$ on one half and $w=Q_{x}$ on the second half, where $P_{x}, Q_{x}$ are the first coordinates of the points found in part (i) of the previous lemma. Then we immediately get $\langle w\rangle_{Q}=\mathbf{w},\left\langle w^{-1 /(p-1)}\right\rangle_{Q}=\mathbf{v}$ and all we need is the $A_{p}$ condition. For any dyadic cube $Q^{\prime}$ properly contained in $Q$, the weight $w$ is constant on $Q^{\prime}$, so

$$
\langle w\rangle_{Q^{\prime}}\left\langle w^{-1 /(p-1)}\right\rangle_{Q^{\prime}}^{p-1}=1 \leq 2 .
$$

It remains to note that on the full cube $Q$ we have

$$
\langle w\rangle_{Q}\left\langle w^{-1 /(p-1)}\right\rangle_{Q}^{p-1}=\mathbf{w v}^{p-1} \leq 2 .
$$

Hence $[w]_{A_{p}} \leq 2$ and the definition of $U$ makes sense.

The second important observation is that the function $U$ does not depend on $Q$. Indeed, for any two dyadic cubes $Q_{1}$ and $Q_{2}$, an affine mapping of one cube onto another puts the classes $A_{p}\left(Q_{1}\right)$ and $A_{p}\left(Q_{2}\right)$ in one-to-one correspondence, and such a change of the variable preserves the averages. 
We will prove that the function $U$ enjoys the following properties.

Theorem 3.2. $\quad$ (i) If $\boldsymbol{y}_{1} \leq \boldsymbol{y}_{2}$, then

$$
U\left(\boldsymbol{x}, \boldsymbol{y}_{1}, \boldsymbol{z}, \boldsymbol{w}, \boldsymbol{v}\right) \leq U\left(\boldsymbol{x}, \boldsymbol{y}_{2}, \boldsymbol{z}, \boldsymbol{w}, \boldsymbol{v}\right) .
$$

(ii) We have

$$
U(\boldsymbol{x}, \boldsymbol{y}, \boldsymbol{z}, \boldsymbol{w}, \boldsymbol{v})=U(\boldsymbol{x}, \boldsymbol{y},|x| \vee \boldsymbol{z}, \boldsymbol{w}, \boldsymbol{v}) .
$$

(iii) We have

$$
U(\boldsymbol{x},|\boldsymbol{x}|,|\boldsymbol{x}|, \boldsymbol{w}, \boldsymbol{v}) \leq 0 .
$$

(iv) We have

$$
U(\boldsymbol{x}, \boldsymbol{y}, \boldsymbol{z}, \boldsymbol{w}, \boldsymbol{v}) \geq \boldsymbol{y} \boldsymbol{w}-K(|\boldsymbol{x}| \vee \boldsymbol{z}) \boldsymbol{w} .
$$

(v) For any $\lambda \neq 0$ and any $\mu>0$ we have

$$
U\left(\lambda \boldsymbol{x},|\lambda| \boldsymbol{y},|\lambda| \boldsymbol{z}, \mu \boldsymbol{w}, \mu^{-1 /(p-1)} \boldsymbol{v}\right)=|\lambda| \mu U(\boldsymbol{x}, \boldsymbol{y}, \boldsymbol{z}, \boldsymbol{w}, \boldsymbol{v}) .
$$

Proof: The condition (i) follows directly from the definition of $U$. To get (ii), simply note that $M f \geq\left|\langle f\rangle_{Q}\right|$ and hence $M f \vee \mathbf{z}=M f \vee$ $|\mathbf{x}| \vee \mathbf{z}$ if $f$ has average $\mathbf{x}$. The condition (iii) follows at once from our assumption (3.1). The fourth property is also easy: it suffices to compute the expression in the definition of $U$ for the constant function $f \equiv \mathbf{x}$. To prove the homogeneity property (3.9), pick arbitrary functions $f, w$ as in the definition of $U(\mathbf{x}, \mathbf{y}, \mathbf{z}, \mathbf{w}, \mathbf{v})$. Then $\lambda f$ has the average $\lambda \mathbf{x}$, and $\mu w$ is an $A_{p}$ weight with characteristics bounded by 2 and such that $\langle\mu w\rangle_{Q}=\mu \mathbf{w},\left\langle(\mu w)^{-1 /(p-1)}\right\rangle_{Q}=\mu^{-1 /(p-1)} \mathbf{v}$. Consequently,

$$
\begin{aligned}
& U\left(\lambda \mathbf{x},|\lambda| \mathbf{y},|\lambda| \mathbf{z}, \mu \mathbf{w}, \mu^{-1 /(p-1)} \mathbf{v}\right) \\
& \geq \frac{1}{|Q|} \int_{Q}\left((|\lambda| \mathbf{y})^{2}-(\lambda \mathbf{x})^{2}+S^{2}(\lambda f)(x)\right)^{1 / 2} \\
& \quad-K M(\lambda f)(x) \vee(|\lambda| \mathbf{z})) \mu w(x) \mathrm{d} x \\
& =|\lambda| \mu \frac{1}{|Q|} \int_{Q}\left[\left(\mathbf{y}^{2}-\mathbf{x}^{2}+S^{2}(f)(x)\right)^{1 / 2}-K(M f(x) \vee \mathbf{z})\right] w(x) \mathrm{d} x .
\end{aligned}
$$

Hence, taking the supremum over all $f$ and $w$, we get

$$
U\left(\lambda \mathbf{x},|\lambda| \mathbf{y},|\lambda| \mathbf{z}, \mu \mathbf{w}, \mu^{-1 /(p-1)} \mathbf{v}\right) \geq|\lambda| \mu U(\mathbf{x}, \mathbf{y}, \mathbf{z}, \mathbf{w}, \mathbf{v}) .
$$

To get the reverse bound, plug in (3.10) the point $(\lambda \mathbf{x},|\lambda| \mathbf{y},|\lambda| \mathbf{z}, \mu \mathbf{w}$, $\left.\mu^{-1 /(p-1)} \mathbf{v}\right)$ in the place of $(\mathbf{x}, \mathbf{y}, \mathbf{z}, \mathbf{w}, \mathbf{v})$ and the numbers $\lambda^{-1}, \mu^{-1}$ in the place of $\lambda$ and $\mu$. 
The main property of the function $U$ is studied in the next statement. It can be regarded as a kind of concavity-type condition.

Theorem 3.3. Let $\alpha=k \cdot 2^{-d}$ for some $k=1,2, \ldots, 2^{d}-1$. Pick $\boldsymbol{x} \in \mathbb{R}$, $\boldsymbol{y} \geq 0, \boldsymbol{z} \geq|\boldsymbol{x}|, \boldsymbol{d}_{ \pm} \in \mathbb{R}$, and $(\boldsymbol{w}, \boldsymbol{v}),\left(\boldsymbol{w}_{ \pm}, \boldsymbol{v}_{ \pm}\right) \in \mathcal{D}$. If

$$
\alpha \boldsymbol{d}_{+}+(1-\alpha) \boldsymbol{d}_{-}=0 \quad \text { and } \quad \alpha\left(\boldsymbol{w}_{+}, \boldsymbol{v}_{+}\right)+(1-\alpha)\left(\boldsymbol{w}_{-}, \boldsymbol{v}_{-}\right)=(\boldsymbol{w}, \boldsymbol{v}) \text {, }
$$

then

$$
\begin{aligned}
U(\boldsymbol{x}, \boldsymbol{y}, \boldsymbol{z}, \boldsymbol{w}, \boldsymbol{v}) \geq & \alpha U\left(\boldsymbol{x}+\boldsymbol{d}_{+}, \sqrt{\boldsymbol{y}^{2}+\boldsymbol{d}_{+}^{2}}, \boldsymbol{z}, \boldsymbol{w}_{+}, \boldsymbol{v}_{+}\right) \\
& +(1-\alpha) U\left(\boldsymbol{x}+\boldsymbol{d}_{-}, \sqrt{\boldsymbol{y}^{2}+\boldsymbol{d}_{-}^{2}}, \boldsymbol{z}, \boldsymbol{w}_{-}, \boldsymbol{v}_{-}\right) .
\end{aligned}
$$

Proof: Pick any functions $f^{+}, w_{+}$and $f^{-}, w_{-}$as in the definitions of $U\left(\mathbf{x}+\mathbf{d}_{+}, \sqrt{\mathbf{y}_{+}^{2}+\mathbf{d}_{+}^{2}}, \mathbf{z}, \mathbf{w}_{+}, \mathbf{v}_{+}\right)$and $U\left(\mathbf{x}+\mathbf{d}_{-}, \sqrt{\mathbf{y}_{-}^{2}+\mathbf{d}_{-}^{2}}, \mathbf{z}, \mathbf{w}_{-}, \mathbf{v}_{-}\right)$, respectively. As we have observed above, the definition of $U$ does not depend on $Q$, so we may assume that these functions are given on a certain fixed dyadic cube $\mathfrak{Q} \subset[0,1)^{d}$ of measure $2^{-d}$. Split $[0,1)^{d}=Q_{+} \cup$ $Q_{-}$, where $Q_{+}$is a disjoint union of $k$ dyadic subcubes of measure $2^{-d}$ each and $Q_{-}$is a disjoint union of $2^{d}-k$ dyadic subcubes of measure $2^{-d}$ each; then $\left|Q_{+}\right|=\alpha$ and $\left|Q_{-}\right|=1-\alpha$. Copy $f^{+}$onto each subcube of $Q_{+}$ and $f^{-}$onto each subcube of $Q_{-}$, and denote the obtained function by $f$. More precisely, if $Q^{\prime} \subseteq Q_{+}$is a cube of measure $2^{-d}$ and $T$ is an affine mapping which sends $Q^{\prime}$ onto $\mathfrak{Q}$, we let $f(x)=f^{+}(T x)$ on $Q^{\prime}$; similarly, if $Q^{\prime} \subseteq Q_{-}$, then let $f(x)=f^{-}(T x)$, where $T$ is as previously. Then $f$ has the average

$$
\langle f\rangle_{[0,1)^{d}}=\int_{Q_{+}} f+\int_{Q_{-}} f=\alpha\left(\mathbf{x}+\mathbf{d}_{+}\right)+(1-\alpha)\left(\mathbf{x}+\mathbf{d}_{-}\right)=\mathbf{x} .
$$

Furthermore, pick a dyadic cube $Q^{\prime} \subseteq Q_{+}$with $\left|Q^{\prime}\right|=2^{-d}$ and let $T: Q^{\prime} \rightarrow \mathfrak{Q}$ be as above. Let us look at the relation between $\left.S(f)\right|_{Q^{\prime}}$, $\left.M(f)\right|_{Q^{\prime}}$, and the corresponding objects for $f^{+}$. Observe that if $x \in Q^{\prime}$, then $f_{0}(x)=\mathbf{x}$ and $f_{n}(x)=f_{n-1}^{+}(T x)$. Consequently, $d f_{0}(x)=\mathbf{x}$, $d f_{1}(x)=\mathbf{d}_{+}$, and $d f_{n}(x)=d f_{n-1}^{+}(T x)$. This implies

$$
S^{2}(f)(x)=\mathbf{x}^{2}+\mathbf{d}_{+}^{2}+S^{2}\left(f^{+}\right)(T x)-\left(\mathbf{x}+\mathbf{d}_{+}\right)^{2} .
$$

Furthermore, since $\left|f_{0}(x)\right|=|\mathbf{x}| \leq \mathbf{z}$, we obtain

$$
M f(x) \vee \mathbf{z}=\sup _{n \geq 1}\left|f_{n}(x)\right| \vee \mathbf{z}=M f^{+}(T x) \vee \mathbf{z} .
$$

When $Q^{\prime} \subseteq Q_{-}$, we have obvious analogues of (3.11) and (3.12): one only needs to change $f^{+}, \mathbf{d}_{+}$to $f^{-}$and $\mathbf{d}_{-}$, respectively. 
Let us apply similar "splicing" procedure to glue $w_{+}$and $w_{-}$into one weight $w$ on $Q$. Arguing as above, one checks that $\langle w\rangle_{Q}=\alpha \mathbf{w}_{+}+$ $(1-\alpha) \mathbf{w}_{-}=\mathbf{w}$ and $\left\langle w^{-1 /(p-1)}\right\rangle_{Q}=\alpha \mathbf{v}_{+}+(1-\alpha) \mathbf{v}_{-}=\mathbf{v}$. Note that $w$ is an $A_{p}$ weight with $[w]_{A_{p}} \leq 2$. Indeed, if $\tilde{Q}$ is a dyadic cube strictly contained in $[0,1)^{d}$, then the product $\langle w\rangle_{\tilde{Q}}\left\langle w^{-1 /(p-1)}\right\rangle_{\tilde{Q}}^{p-1}$ is equal to the corresponding product for $w_{+}$or $w_{-}$(depending on whether $\tilde{Q} \subseteq Q_{+}$ or $\tilde{Q} \subseteq Q_{-}$), so it is smaller than 2 ; on the other hand, as we have just verified,

$$
\langle w\rangle_{[0,1)^{d}}\left\langle w^{-1 /(p-1)}\right\rangle_{[0,1)^{d}}^{p-1}=\mathbf{w v}^{p-1} \leq 2 .
$$

Now let us check how the above relations between $f_{ \pm}, f, w_{ \pm}$, and $w$ affect the special function $U$. We have

$$
\begin{aligned}
& U(\mathbf{x}, \mathbf{y}, \mathbf{z}, \mathbf{w}, \mathbf{v}) \\
& \geq \int_{[0,1)^{d}}\left[\left(\mathbf{y}^{2}-\mathbf{x}^{2}+S^{2}(f)(x)\right)^{1 / 2}-K(M f(x) \vee \mathbf{z})\right] w(x) \mathrm{d} x \\
& =\frac{\alpha}{\left|Q_{+}\right|} \int_{Q_{+}}\left[\left(\mathbf{y}^{2}-\mathbf{x}^{2}+S^{2}(f)(x)\right)^{1 / 2}-K(M f(x) \vee \mathbf{z})\right] w(x) \mathrm{d} x \\
& \quad+\frac{1-\alpha}{\left|Q_{-}\right|} \int_{Q_{-}}\left[\left(\mathbf{y}^{2}-\mathbf{x}^{2}+S^{2}(f)(x)\right)^{1 / 2}-K(M f(x) \vee \mathbf{z})\right] w(x) \mathrm{d} x .
\end{aligned}
$$

Let us look at the first summand on the right hand side. Pick $Q^{\prime} \subset Q_{+}$ of measure $2^{-d}$. By (3.11) and (3.12), we have

$$
\begin{aligned}
& \frac{1}{\left|Q^{\prime}\right|} \int_{Q^{\prime}}\left[\left(\mathbf{y}^{2}-\mathbf{x}^{2}+S^{2}(f)(x)\right)^{1 / 2}-K(M f(x) \vee \mathbf{z})\right] w(x) \mathrm{d} x \\
& =\frac{1}{|\mathfrak{Q}|} \int_{\mathfrak{Q}}\left[\left(\mathbf{y}^{2}+\mathbf{d}_{+}^{2}-\left(\mathbf{x}+\mathbf{d}_{+}\right)^{2}+S^{2}\left(f^{+}\right)(x)\right)^{1 / 2}-K\left(M f^{+}(x) \vee \mathbf{z}\right)\right] w_{+}(x) \mathrm{d} x
\end{aligned}
$$

and hence

$$
\begin{aligned}
& \frac{\alpha}{\left|Q_{+}\right|} \int_{Q_{+}}\left[\left(\mathbf{y}^{2}-\mathbf{x}^{2}+S^{2}(f)(x)\right)^{1 / 2}-K(M f(x) \vee \mathbf{z})\right] w(x) \mathrm{d} x \\
& =\frac{\alpha}{|\mathfrak{Q}|} \int_{\mathfrak{Q}}\left[\left(\mathbf{y}^{2}+\mathbf{d}_{+}^{2}-\left(\mathbf{x}+\mathbf{d}_{+}\right)^{2}+S^{2}\left(f^{+}\right)(x)\right)^{1 / 2}-K\left(M f^{+}(x) \vee \mathbf{z}\right)\right] w_{+}(x) \mathrm{d} x .
\end{aligned}
$$

We handle the integral over $Q_{-}$exactly in the same manner. It suffices to take the supremum over all $f^{ \pm}, w_{ \pm}$as above to get the claim. 
We are ready for the proof of Theorem 1.3. We will sometimes use the following notation: if $\mathbf{x} \in \mathbb{R}, \mathbf{y}, \mathbf{z} \geq 0$, and $P=(\mathbf{w}, \mathbf{v}) \in \mathcal{D}$, we will write $U(\mathbf{x}, \mathbf{y}, \mathbf{z} ; P):=U(\mathbf{x}, \mathbf{y}, \mathbf{z}, \mathbf{w}, \mathbf{v})$. We start from the observation that, by Theorem 3.2(i) and (iii),

$$
0 \geq U\left(1,1,1,1,2^{1 /(p-1)}\right) \geq U\left(1,0,1,1,2^{1 /(p-1)}\right) .
$$

Next, we use Theorem 3.3: by (3.4), we obtain that for each $k$,

$$
\begin{aligned}
U\left(1,(2 k)^{1 / 2}, 1 ; T_{k}\right) \geq & 2^{-d} U\left(2^{d},\left(2 k+\left(2^{d}-1\right)^{2}\right)^{1 / 2}, 1 ; R\right) \\
& +\left(1-2^{-d}\right) U\left(0,(2 k+1)^{1 / 2}, 1 ; T_{k+1}\right) .
\end{aligned}
$$

By part (ii) of Theorem 3.2, this expression is equal to

$$
\begin{aligned}
2^{-d} U\left(2^{d},\left(2 k+\left(2^{d}-1\right)^{2}\right)^{1 / 2}, 2^{d}\right. & ; R) \\
& +\left(1-2^{-d}\right) U\left(0,(2 k+1)^{1 / 2}, 1 ; T_{k+1}\right),
\end{aligned}
$$

which by parts (i) and (v), is not smaller than

$$
\begin{aligned}
& 2^{-d} U\left(2^{d}, 0,2^{d} ; R\right)+\left(1-2^{-d}\right) U\left(0,(2 k+1)^{1 / 2}, 1 ; T_{k+1}\right) \\
& \quad \geq R_{x} U\left(1,0,1,1,2^{1 /(p-1)}\right)+\left(1-2^{-d}\right) U\left(0,(2 k+1)^{1 / 2}, 1 ; T_{k+1}\right) .
\end{aligned}
$$

Furthermore, by Theorem 3.3 and Theorem 3.2(v) again,

$$
\begin{aligned}
U\left(0,(2 k+1)^{1 / 2}, 1 ; T_{k+1}\right) \geq & \frac{1}{2} U\left(-1,(2 k+2)^{1 / 2}, 1 ; T_{k+1}\right) \\
& +\frac{1}{2} U\left(1,(2 k+2)^{1 / 2}, 1 ; T_{k+1}\right) \\
= & U\left(1,(2 k+2)^{1 / 2}, 1 ; T_{k+1}\right) .
\end{aligned}
$$

Hence, by induction, we obtain

$$
\begin{aligned}
U\left(1,0,1,1,2^{1 /(p-1)}\right)= & U\left(1,0,1 ; T_{0}\right) \\
\geq & \left(1-2^{-d}\right)^{N} U\left(1,(2 N)^{1 / 2}, 1 ; T_{N}\right) \\
& +\sum_{k=0}^{N-1}\left(1-2^{-d}\right)^{k} R_{x} U\left(1,0,1,1,2^{1 /(p-1)}\right) \\
= & \left(1-2^{-d}\right)^{N} T_{x} U\left(1,(2 N)^{1 / 2}, 1,1,1\right) \\
& +\left(1-\left(1-2^{-d}\right)^{N}\right) 2^{d} R_{x} U\left(1,0,1,1,2^{1 /(p-1)}\right) .
\end{aligned}
$$


Now we assume that $d$ is large; if we apply (3.3) and (3.13), we obtain $U\left(1,(2 N)^{1 / 2}, 1,1,1\right) \leq \frac{1-\left(1-\left(1-2^{-d}\right)^{N}\right) 2^{d} R_{x}}{\left(1-2^{-d}\right)^{N} T_{x}} U\left(1,0,1,1,2^{1 /(p-1)}\right) \leq 0$.

This, by (3.8), implies $\sqrt{2 N}-K \leq 0$. This is a contradiction, since $N$ was arbitrary. The claim is proved.

\section{Acknowledgments}

The author would like to thank an anonymous referee for a very careful reading of the paper and many helpful suggestions, which greatly improved the presentation.

\section{References}

[1] S. M. BuCKLEY, Estimates for operator norms on weighted spaces and reverse Jensen inequalities, Trans. Amer. Math. Soc. 340(1) (1993), 253-272. DOI : $10.2307 / 2154555$.

[2] D. L. Burkholder, Martingale transforms, Ann. Math. Statist. 37(6) (1966), 1494-1504. DOI: 10.1214/aoms/1177699141.

[3] D. L. Burkholder, The best constant in the Davis inequality for the expectation of the martingale square function, Trans. Amer. Math. Soc. 354(1) (2002), 91-105. DOI: 10.1090/S0002-9947-0102887-2.

[4] E. A. Carlen And P. KrÉE, On martingale inequalities in noncommutative stochastic analysis, J. Funct. Anal. 158(2) (1998), 475-508. DOI: 10.1006/jfan.1998.3299.

[5] D. Cruz-Uribe, J. M. Martell, and C. PÉrez, Sharp weighted estimates for classical operators, Adv. Math. 229(1) (2012), 408-441. DOI : 10.1016/j . aim.2011.08.013.

[6] B. DAvis, On the intergrability of the martingale square function, Israel J. Math. 8(2) (1970), 187-190. DOI: $10.1007 /$ BF02771313.

[7] C. Dellacherie And P.-A. Meyer, "Probabilities and potential. B. Theory of martingales", Translated from the French by J. P. Wilson, North-Holland Mathematics Studies 72, North-Holland Publishing Co., Amsterdam, 1982.

[8] O. Dragičević, L. Grafakos, M. C. Pereyra, and S. PeTERMICHL, Extrapolation and sharp norm estimates for classical operators on weighted Lebesgue spaces, Publ. Mat. 49(1) (2005), 73-91. DOI : 10.5565/PUBLMAT_49105_03. 
[9] R. F. Gundy and R. L. Wheeden, Weighted integral inequalities for the nontangential maximal function, Lusin area integral, and Walsh-Paley series, Studia Math. 49 (1973/74), 107-124.

[10] S. Hukovic, S. Treil, And A. VolberG, The Bellman functions and sharp weighted inequalities for square functions, in: "Complex Analysis, Operators, and Related Topics", Oper. Theory Adv. Appl. 113, Birkhäuser, Basel, 2000, pp. 97-113.

[11] A. Khintchine, Über dyadische Brüche, Math. Z. 18(1) (1923), 109-116. DOI: $10.1007 / \mathrm{BF} 01192399$.

[12] A. K. Lerner, On some sharp weighted norm inequalities, $J$. Funct. Anal. 232(2) (2006), 477-494. DOI: 10.1016/j.jfa.2005. 08.006 .

[13] A. K. LERNER, On some weighted norm inequalities for LittlewoodPaley operators, Illinois J. Math. 52(2) (2008), 653-666.

[14] J. E. LitTLEWOOD, On bounded bilinear forms in an infinite number of variables, Quart. J. Math. Oxford Ser. 1 (1930), 164-174. DOI: $10.1093 /$ qmath/os-1.1.164.

[15] J. MarCinkiEWicz, Quelques théorèmes sur les séries orthogonales, Ann. Soc. Polon. Math. 16 (1937), 84-96.

[16] J. Marcinkiewicz AND A. Zygmund, Quelques théorèmes sur les fonctions indépendantes, Studia Math. 7 (1938), 104-120.

[17] F. L. Nazarov and S. R. Treil, The hunt for a Bellman function: applications to estimates for singular integral operators and to other classical problems of harmonic analysis, St. Petersburg Math. J. 8(5) (1997), 721-824.

[18] F. Nazarov, S. Treil, ANd A. Volberg, The Bellman functions and two-weight inequalities for Haar multipliers, J. Amer. Math. Soc. 12(4) (1999), 909-928. DOI: 10.1090/S0894-0347-99-00310-0.

[19] R. E. A. C. PALEY, A remarkable series of orthogonal functions (I), Proc. London Math. Soc. S2-34(1), 241-264. DOI: 10.1112/plms/ s2-34.1.241.

[20] S. Petermichl and S. Pott, An estimate for weighted Hilbert transform via square functions, Trans. Amer. Math. Soc. 354(4) (2002), 1699-1703. DOI: 10.1090/S0002-9947-01-02938-5.

[21] G. Pisier and Q. XU, Non-commutative martingale inequalities, Comm. Math. Phys. 189(3) (1997), 667-698. DOI: 10.1007/s0022 00050224.

[22] E. M. Stein, The development of square functions in the work of A. Zygmund, Bull. Amer. Math. Soc. (N.S.) 7(2) (1982), 359-376. DOI : $10.1090 /$ S0273-0979-1982-15040-6. 
[23] E. M. Stein, "Harmonic Analysis: Real-variable Methods, Orthogonality, and Oscillatory Integrals", With the assistance of Timothy S. Murphy, Princeton Mathematical Series 43, Monographs in Harmonic Analysis III, Princeton University Press, Princeton, NJ, 1993.

[24] V. I. VAsyunin, The sharp constant in the reverse Hölder inequality for Muckenhoupt weights (Russian), Algebra $i$ Analiz 15(1) (2003), 73-117; translation in St. Petersburg Math. J. 15(1) (2004), 49-79. DOI : 10.1090/S1061-0022-03-00802-1.

[25] M. Wilson, "Weighted Littlewood-Paley Theory and Exponentialsquare Integrability", Lecture Notes in Mathematics 1924, Springer, Berlin, 2008.

[26] J. Wittwer, A sharp estimate on the norm of the continuous square function, Proc. Amer. Math. Soc. 130(8) (2002), 2335-2342. DOI : $10.1090 /$ S0002-9939-02-06342-6.

Department of Mathematics, Informatics and Mechanics University of Warsaw

Banacha 2

02-097 Warsaw

Poland

E-mail address: ados@mimuw.edu.pl

Primera versió rebuda el 29 de març de 2016, darrera versió rebuda el 4 de novembre de 2016. 